\title{
A procedure for the analysis of intrinsic facial form and growth
}

\author{
An equivalent-balance concept
}

\author{
Donald H. Enlow, Ph.D., Robert E. Moyers, D.D.S., Ph.D., \\ W. Stuart Hunter, D.D.S., Ph.D., and James A. McNamara, Jr., D.D.S., M.S. \\ Ann Arbor, Mich., and London, Ontario, Canada
}

$\mathrm{T}_{\mathrm{hi}}$ his report introduces and describes a method of cephalometric evaluation for individuals based on their own particular morphologic and morphogenetic facial patterns. References to statistical population standards are not required. The purpose of the procedure is to analyze the nature of anatomic fit among the different bones of the craniofacial complex of one subject at any age and through time. The method is presented in two parts: (1) a "form analysis" which deals with facial pattern, construction, and dimensions and (2) a "growth analysis" which is concerned with an interpretation of incremental changes. This procedure is based on four basic morphologic concepts which are summarized below.

The concept of architectural equivalence. In any functional assemblage of bones, such as the craniofacial complex, certain key dimensions must necessarily correspond between these bones in order to provide proper fit. A preseribed portion of each bone represents a direct architectural (dimensional) counterpart of some segment of another bone (or bones), even though their respective functions and other anatomic relationships are different. These dimensional analogues among bones are termed "equivalents.", "If any two such equivalents match (or nearly match), a dimensional balance is thereby produced and morphologic fit is provided. If a match does not occur, however, corresponding architectural imbalance results and affects not only their own fit but that of other contiguous bones as well. As will be seen, an analysis of the nature of growth increments is a mcaningful consideration, since continued growth can (1)

From the Department of Anatomy, The University of Miehigan (Drs. Enlow and MeNamara); The University of Miehigan Center for Human Growth and Development (Drs. Enlow, Moyers, and Hunter); and the Department of Paediatric Dentistry, The University of Western Ontario (Dr. Hunter).

This study was supported, in part, by United States Public Hoalth Service Grant DE-02272. 
sustain a balanced or an imbalanced condition, (2) improve an unbalanced situation, or (3) aggravate the original status of balance or imbalance (Fig. 1).

Although a large number of different architectural equivalents exists throughout the skull as a whole, only a few (Fig. 2) are relevant to the purpose of the present analysis.

The concept of effective dimensions. In order to equate any two bony "equivalents," only those portions that represent an actual structural and dimensional counterpart can be considered. The entire length or width of a bone is not ordinarily involved. Rather, only that particular span that provides proper fit to a corresponding segment of another bone is relevant. For example, the horizontal bony arch of the mandible is a direct structural equivalent to the bony maxillary arch. The ramus portion of the mandible, on the other hand, is not directly involved since this segment of the mandible provides structural equivalence to another entirely different part of the skull, that is, a part of the cranial floor. Similarly, the entire cranial fioor is not directly involved in maintaining architectural equivalence to parts of the face but only those portions actually in direct structural and dimensional balance to the facial parts. In order to apply the concept of equivalence to cephalometric analyses, it is thus essential to identify and accurately define the particular effective dimensions that constitute equivalence in any given composite of bones. This is an important consideration. In the present study, the basic plan of plane orientation, the selection of landmarks, and the evaluation of both pattern and growth are all dependent on this principle.

The concept of aggregate balance. Any two architectural equivalents ordinarily occur as members of a composite group comprising several different sets

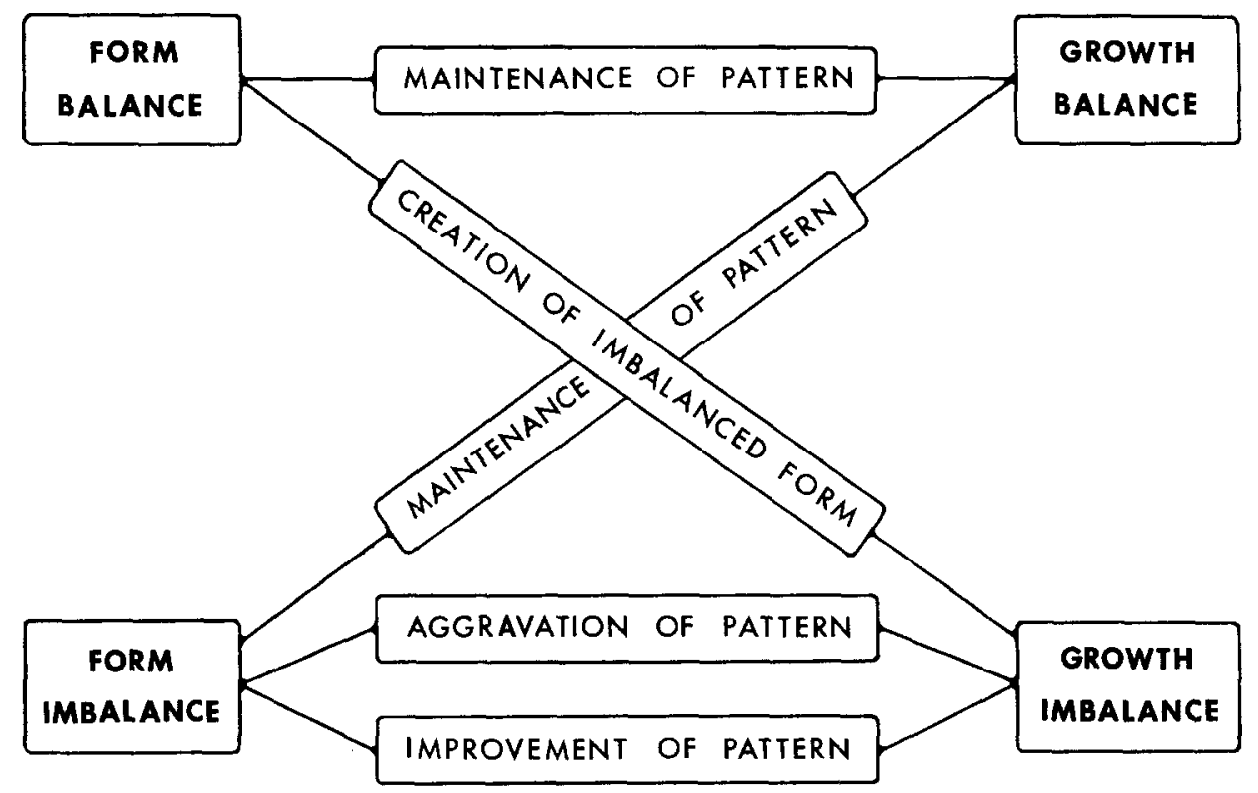

Fig. 1. Form and growth relationships between architectural equivalents. 
of such structural counterparts. Because somatic and genetic variations are regularly present in the structure of the bones involved, a factor of reciprocal adjustment ean occur in some of their dimensions for mutual accommodation of variations in size and shape. For example, the sum of bony mandibular arch and ramus horizontal dimensions should closely balance the sum of bony maxillary arch and "effective" cranial floor dimensions. A number of different combinations are possible, however, to achieve composite balance among all of them (Fig. 2). Thus, the cranial floor may be "long" relative to its direct equivalent,
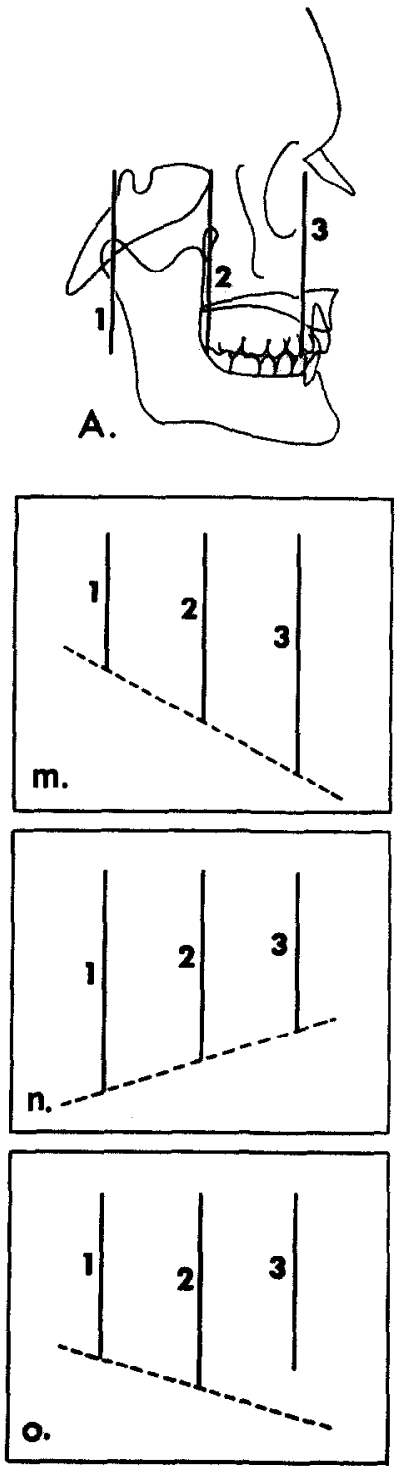
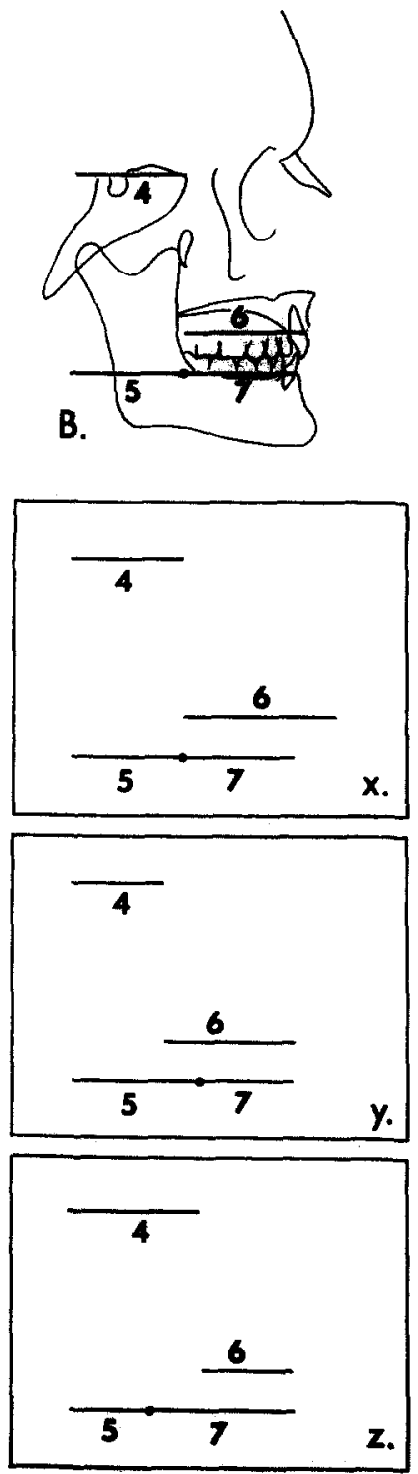

Fig. 2. For legend, see opposite page. 
the ramus. This regional dimensional imbalance, however, can be offset by a corresponding imbalance in another set of equivalents, thereby providing adjustment when aggregate balance between the sum of all of them is considered. "Imbalances" in some individual dimensions can therefore mutually compensate for each other relative to their over-all composite pattern.

The concept of incremental balance. The significance of growth changes in the different bones of the craniofacial complex can be evaluated in a more meaningful way if interpreted on the basis of the three concepts outlined above. If only growth increments are considered, without an analysis of the form and dimensional balance at the ages involved, a misleading picture could result. Aggregate balance can change through time by correcting (or improving) or by aggravating an original dimensional balance or imbalance between regional equivalents. The "form analysis" reveals where imbalance exists and to what extent. The "growth analysis" discloses how this original balance is either sustained or changed as a consequence of continued growth (and/or treatment).

Thus, the concept of "balance" applies to increments of growth as well as to effective anatomic dimensions among the structural equivalents. If any two equivalents dimensionally match each other, they are in balance. If this condition is sustained during continued growth, the incremental increases are similarly balanced. If a dimensional imbalance exists, an incremental imbalance can subsequently take place in such a way that dimensions are later brought into actual balance. Dimensional balance at an early age, however, can become upset by imbalanced growth increments between equivalents. It is necessary, there-

Fig. 2. Diagram A illustrates the three vertical architectual equivalents included in this study: the cranial floor-ramus vertical (1); the posterior nasomaxilla (2); and the anterior nasomaxilla (3). As shown in A, they are all in exact dimensional balance, and the functional occlusal plane therefore coincides with the neutral occlusal axis, which is perpendicular to these three vertical planes. If vertical dimensional imbalance occurs, however, downward occlusal rotation (m), upward occlusal rotation (n), or open-bite (0) necessarily results.

Diagram B illustrates the four horizontal architectural equivalents included in the present analysis; the cranial floor (4), the ramus (5), the maxilla (6), and the mandibular corpus (7). In all equivalents (vertical as well as horizontal), only the effective dimension of each bone is utilized. If these horizontal equivalents are balanced, as in B, their effective dimensions are very close to an exact match and the bones "fit" relative to each other. Diagram $x$ shows a maxillary equivalent that is excessive relative to a disproportionately smaller corpus. The other equivalents (4 and 5) are balanced. The result is maxillary protrusion. Diagram y demonstrates a similar maxillary-mandibular imbalance, but the ramus, although actually out of balance to the cranial base, serves to provide dimensional compensation so that aggregate balance is achieved in the over-all composite of equivalents. Diagram z illustrates a "long" cranial floor that is not dimensionally in balance with a "short" ramus. However, aggregate balance is produced by reciprocal adjustment between the corpus and the maxilla, so that the sum of all their dimensions has been balanced. It is apparent that many other similar combinations are possible among the composites of these architectural equivalents which will produce either a balanced or an imbalanced face. 
fore, to consider balance of effective dimensions among individual sets of equivalents at all ages and to evaluate the aggregate balance among them; then the significance of growth changes between the different ages can be more meaningfully interpreted.

\section{Planes and landmarks}

Most of the traditional cephalometric landmarks and planes are not appropriate or usable in the present analysis, since the procedure is based on an evaluation of effective dimensions between specific anatomic equivalents of structure. Therefore, there has been defined a simple series of planes that

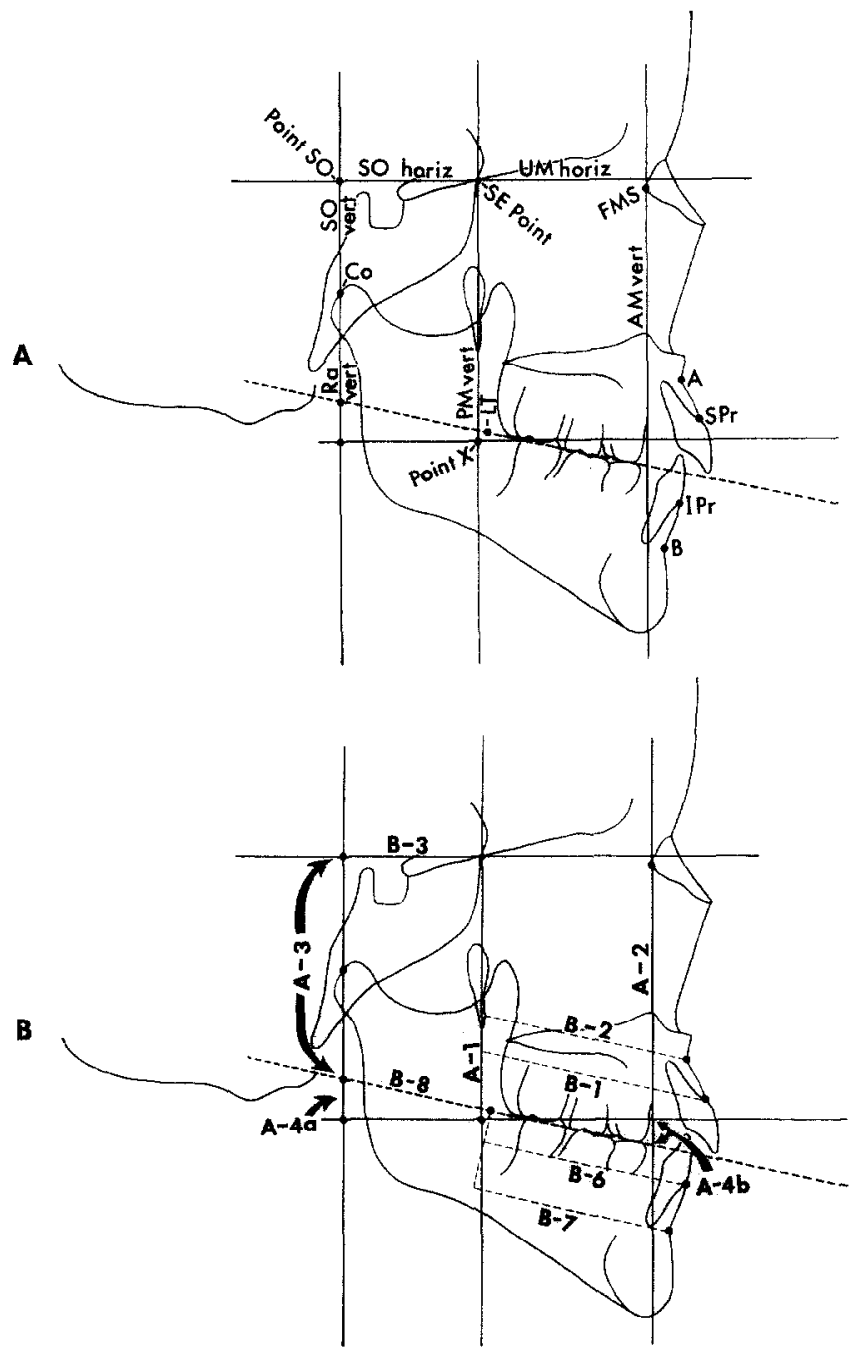

Fig. 3. The landmarks, points, and planes utilized in this study are summarized in $\mathbf{A}$. (See text for description of each.) The code used in B represents the vertical and horizontal measurements along the various planes. This code corresponds to the itemization in the "Outline of Procedure" presented in the text and also to the itemization in Tables I and II. 
serves to delineate these particular equivalent dimensions (Fig. 3). Their primary purpose is to enable one to determine the nature of actual fit among the different major bones and parts of bones. These planes identify three vertical and four horizontal equivalent dimensions. From these, the effective height of the anterior portion of the nasomaxillary complex can be equated with its posterior height. Both, in turn, can be analyzed for their match with the effective ramus-cranial floor composite height. Similarly, the horizontal length of the bony maxillary arch can be equated with the mandibular arch, and the match and balance of the effective horizontal dimension of the cranial floor can be compared with the horizontal (longitudinal) breadth of the ramus. In most instances, the entire dimensions of the bones are not involved, since the planes themselves are constructed in such a way that only the effective dimensions of each bone are utilized. Significantly, these planes conform naturally with major sites of growth, remodeling, and displacement, including the condyle, maxillary tuberosity, anterior alveolar edges, occlusal plane, palate, frontomaxillary suture, ramus-carpus transition, ethmofrontal suture, and the synchondroses.

Landmarks and points. The landmarks used are bilateral, but it is recommended that they be averaged between right and left. One should not, however, average some but not others, since consistency is required for accuracy.

$S E$ point (spheno-ethmoidal). This point is determined by the intersection of the great wing of the sphenoid and the cranial floor as seen in lateral head films. The purpose is to represent the spheno-ethmoidal junction, which is located slightly posterior to this intersection. The spheno-ethmoidal (and the continuous sphenofrontal) junction separates that part of the cranial floor associated directly with the underlying maxilla from the portion associated with the ramus and postmaxillary pharyngeal space. The posterior surface of the maxillary tuberosity characteristically aligns with this juncture. This anatomic feature is utilized as a key relationship for the construction of the basic horizontal and vertical planes drawn on the head film tracings.

FMS point (frontomaxillary suture). This is the superiormost point of the suture at its articulation with the nasal and frontal bones. The purpose is to identify a relevant point for determining the effective vertical height of the anterior nasomaxillary complex. Either the anterior or posterior corner of the suture may be utilized, as desired, but one or the other should be used with consistency between the serial head films.

Co (condylion). The most posterior-superior point on the mandibular condylc, this point is used to establish the effective horizontal and vertical dimensions of both the ramus and the cranial floor.

Condylion can usually be found, although at times with difficulty. Articulare (intersection of the posterior edge of the ramus and the ventral surface of the occipital) may be substituted, if necessary, but this should be done with consistency between serial head films. Although the use of Ar will prevent an analysis of individual ramus and cranial base heights, respectively, this distinction is not ordinarily required, since the composite height is sufficient for the present purpose. (Note: A distinction between their horizontal dimensions is required, however, as described elsewhere.)

SO point (spheno-occipital). The intersection of the SO vertical and hori- 
zontal planes (see below), SO point is used to establish the effective vertical and horizontal dimensions of the cranial floor.

PNS (posterior nasal spine). PNS is the most posterior point on the sagittal plane of the hard palate. It is used to distinguish the nasal from the alveolar portions of the nasomaxillary complex. (Note: The present study has not been extended to a differential analysis between these two regions.)

A point (subspinale). The most posterior point on the anterior shadow of the maxilla above the central incisor, " $A$ " point is used to determine the "basal" length of the bony arch exclusive of the more labile alveolar protrusion.

$B$ point (supramentale). The most posterior point on the anterior surface of the symphyseal outline, " $B$ " point is used to measure the basal length of the mandibular corpus exclusive of the alveolar process and mental protuberance.

$S P r$ point (superior prosthion). As used in this study, SPr is the point of contact between the alveolar margin and the two central maxillary ineisors.

$I P r$ (or ID) point (inferior prosthion; infradentale). This is the point of contact between the alveolar process and the two central incisors of the mandible.

$X$ point. The intersection of the PM vertical plane and the neutral occlusal axis (see below), $\mathrm{X}$ point is used to establish the over-all effective height of the posterior nasomaxillary complex. It is also utilized to determine the direction and the extent of any ocelusal rotation present.

LT point (lingual tuberosity). This point represents an equivalent of the posterior surface of the maxillary tuberosity (as identified by PTM). LT point is used to determine the horizontal dimensions of both the corpus and the ramus. It is located by determining the horizontal dimension of the ramus from its anterior to posterior margins along the functional occlusal plane (see below). This same dimension is then extended anteriorly from the intersection of the occlusal plane and the Ra vertical lines (dividers may be conveniently used for this purpose). The resulting point is the effective location of the lingual tuberosity relative to the breadth and angle of the ramus. (Note: The LT point represents the sizable lingual tuberosity and not the "lingula," which is a diminutive bony projection overlying the mandibular foramen. The lingual tuberosity itself is the medial protruding housing for the last molar and is thus a structural counterpart of the maxillary tuberosity.)

Unlike PTM, this landmark does not have a clear-cut margin and cannot be visualized in head films. Its effective location must, therefore, be calculated as described above. The ramus and the corpus characteristically overlap, so that the ramus extends anteriorly for a distance well beyond the posterior limit of the corpus (the lingual tuberosity). However, the ramus is obliquely disposed, and the distance of ramus-corpus overlap is offset and approximately equaled by the distance from the posterior edge of the ramus to the Ra vertical plane (see below). The result is a squaring effect of the ramus, so that its "effective" over-all breadth equals the distance from $L T$ point to the perpendicular $R a$ vertical plane. Note that the distance from the posterior margin of the ramus to the $\mathrm{Ra}$ vertical line equals the distance from the anterior margin of the ramus to the LT point. If the various horizontal equivalents are balanced, the LT point 
will lie very close to the PM vertical line and to the $\mathrm{X}$ point. If a dimensional imbalance exists in this region, however, the LT point may lie as much as 5 or $6 \mathrm{~mm}$. to either side of the PM plane.

\section{Planes and axes}

$P M$ vertical (posterior nasomaxilla). This is a line drawn inferiorly from SE point along the posterior surface of the maxillary tuberosity.* For serial consistency, the line is regularly passed through the inferior- and posteriormost point of PTM (Fig. 3). The PM vertical plane is used to determine the verlical dimension of the posterior nasomaxillary complex, and it also serves to delineate the effective horizontal dimensions of the maxillary body and the cranial floor posterior to it. This important plane should be the first construction line placed on the tracing, since most of the other planes and lines are then drawn either perpendicular or parallel to it.

$U M$ horizontal (upper maxilla). This is a perpendicular to PM vertical extending anteriorly from SE point.

$A M$ vertical (anterior nasomaxilla). This is a line drawn inferiorly from FMS point parallel to PM vertical. The measurement from the intersection of this line with the UM horizontal line down to the functional occlusal plane (see below) represents the effective (not over-all) height of the anterior maxilla.

SO horizontal (spheno-occipital). A perpendicular line to $\mathrm{PM}$ vertical extending posteriorly from SE point to SO point, this defines the effective horizontal dimension of the cranial base relative to its equivalent, the ramus.

SO vertical. This is a perpendicular line from SO horizontal (parallel with PM vertical) extending inferiorly from SO point to condylion. It is the effective (not over-all) height of the cranial floor. (Note: Condylion is the determining landmark used; SO point is a result of the plane intersection.)

$R a$ vertical (ramus). An inferior extension of the $\mathrm{SO}$ vertical line from condylion down to the functional and neutral occlusal planes, this identifics the effective (not over-all) height of the ramus relative to the vertical dimensions of the maxilla.

Neutral occlusal axis (NOA). This is a perpendicular from PM vertical extending anteriorly through the posterior- and inferiormost maxillary molar contact point. Only a fully crupted molar is to be used. If the last molar, although erupted, is situated higher than the occlusal plane common to the other maxillary molars and premolars, the next-to-last molar should be used. The purpose of this plane is to establish the maximum effective height of the posterior portion of the nasomaxillary complex. If the last molar were to be utilized when in a high position, both a false measurement and an inaccurate occlusal plane determination would result.

Functional occlusal plane (FOP). This is a line drawn anteriorly from the inferior posteriormost maxillary-mandibular contact point (as explained above) to the maxillary-mandibular contact point of that tooth situated on the AM vertical line. The incisors are not considered in the construction of this line;

* Not to be confused with the Bimler vertical line, which is based on Frankfort plane.1, 2 
therefore, it does not represent the occlusal plane as customarily defined. ${ }^{5}$ It will be found that the "functional" and "neutral" occlusal lines coincide (or nearly do) in many faces that have balanced dimensional equivalents. If some vertical imbalance exists, however, either an upward or a downward occlusal rotation results, and the functional and neutral planes will diverge to a corresponding degree. A relatively moderate degree of such divergence is not, in itself, of direct significance if the nature of the imbalance is proportionate between the three vertical planes and bimaxillary occlusion is attained without anterior or posterior open-bite. The presence of a vertical imbalance, however, can predispose occlusal problems, since reciprocal adjustments are necessarily required for compensation. Also, more severe cases of rotation have been found to be associated, at least indirectly, with skeletal imbalances that are essentially of a horizontal rather than a vertical nature.

If the functional and the neutral occlusal planes approximately coincide, gonion is generally situated about halfway between the PM and the Ra vertical lines. If a marked degree of downward occlusal rotation has occurred, gonion is usually located more toward the Ra vertical line. Conversely, if upward rotation has taken place, it is positioned somewhat more toward the PM vertical

Table I. Form analysis

A. Vertical

1. Posterior maxilla (PM vertical)

2. Anterior maxilla (AM-UM intersection to functional occlusal plane)

3. Composite ramus-cranial floor (Ra-SO verticals)

a. cranial floor (SO vertical) if desired

b. ramus ( $\mathrm{Ra}$ vertical) if desired

4. Functional occlusal plane divergence from neutral axis

a. measured at Ra vertical

b. measured at AM vertical

5. Mandibular-maxillary contact plane divergence from each other (if premolar open-bite exists)

6. Maxillary-mandibular incisor relationships

7. Commentary on vertical balance: (See later discussion)

B. Horizontal

1. Nasomaxilla (Pr to PM vertical)

2. Nasomaxilla (A point to PM vertical)

3. Cranial floor (\$O horizontal)

4. Total $(1+3)$

5. Total $(2+3)$

6. Mandibular corpus (IPr to LT)

7. Mandibular corpus (B point to LT)

8. Ramus (LT to Ra vertical)

9. Total $(6+8)$

10. Total $(7+8)$

11. Commentary on horizontal balance: (See later discussion) 
line. The ramus-to-corpus angle itself is essentially related to the vertical dimension of the PM vertical plane. As this dimension lengthens, the ramus becomes more upright and the angle correspondingly decreases.

Maxillary and mandibular conlacl planes. If a vertical dimensional imbalance has produced ocelusal rotation, either up or down, so that the functional plane not only diverges from the neutral ocelusal axis but the maxillary contact plane also diverges from the mandibular contact plane, it is then necessary to identify them separately. A line is drawn from their posteriormost contact point(s) to the contact points (not cusp tips) of corresponding teeth that would be situated on the AM vertical line if occlusion were closed. These lines are then extended back to the Ra vertical line in order to calculate the extent of their respective divergence relative to the ramus.

$C F$ axis (cranial floor). A line that joins FMS point with SE point and extends posteriorly, this plane has not been utilized in the present analysis but is being included in continuing studies dealing with cranial floor rotation. It has been observed that this plane closely parallels the palatal plane, and any rotation of one is generally accompanied by similar rotation of the other.

\begin{tabular}{|c|c|c|c|c|c|c|c|}
\hline \multicolumn{2}{|c|}{ Subject $A$} & \multicolumn{2}{|c|}{ Subject $B$} & \multicolumn{4}{|c|}{ Subject $C$} \\
\hline $\begin{array}{l}\text { ryrs. } \\
(\mathrm{mm} .)\end{array}$ & $\begin{array}{c}14 \text { yrs. } \\
(\mathrm{mm} .)\end{array}$ & $\begin{array}{l}7 \text { yrs. } \\
(m m .)\end{array}$ & $\begin{array}{c}14 \text { yrs. } \\
\text { (mm.) }\end{array}$ & $\begin{array}{l}8 \text { yrs. } \\
\text { (mm.) }\end{array}$ & $\begin{array}{c}10 \text { yrs. } \\
(\mathrm{mm} .)\end{array}$ & $\begin{array}{c}12.6 \text { yrs. } \\
(\mathrm{mm} .)\end{array}$ & $\begin{array}{c}14.6 \text { yrs. } \\
(\mathrm{mm} .)\end{array}$ \\
\hline 62.7 & 78.3 & 59.4 & 75.9 & 56.4 & 60.1 & 65.0 & 65.6 \\
\hline 62.0 & 77.7 & 64.8 & 78.1 & 58.6 & 61.8 & 66.6 & 68.8 \\
\hline 67.4 & 80.3 & 51.5 & 71.4 & 52.3 & 56.3 & 61.9 & 61.4 \\
\hline 4.7 & 2.0 & 7.9 & 4.5 & 4.1 & 3.7 & 3.2 & 4.2 \\
\hline 0.7 & 0.8 & 5.2 & 2.0 & 2.0 & 1.7 & 1.8 & 3.1 \\
\hline 0 & 0 & 0 & 0 & 0 & 0 & 0 & 0 \\
\hline
\end{tabular}

\begin{tabular}{llrrrrrr}
55.4 & 60.4 & 51.5 & 63.8 & 53.0 & 57.2 & 59.2 & 62.0 \\
52.2 & 54.9 & 48.7 & 58.4 & 50.8 & 53.5 & 55.5 & 56.7 \\
35.5 & 39.0 & 32.9 & 40.2 & 32.5 & 32.8 & 34.4 & 35.8 \\
90.9 & 99.4 & 84.4 & 104.0 & 85.5 & 90.0 & 93.6 & 97.8 \\
87.7 & 93.9 & 81.6 & 98.6 & 83.3 & 86.3 & 89.9 & 92.5 \\
53.1 & 58.4 & 42.7 & 59.1 & 49.4 & 50.0 & 50.0 & 57.3 \\
52.1 & 56.4 & 42.1 & 55.2 & 47.7 & 49.1 & 47.9 & 56.0 \\
36.1 & 40.5 & 33.7 & 39.6 & 35.7 & 38.0 & 40.8 & 38.3 \\
89.2 & 98.9 & 76.4 & 98.7 & 85.1 & 88.0 & 90.8 & 95.6 \\
88.2 & 96.9 & 75.8 & 94.8 & 83.4 & 87.1 & 88.7 & 94.3 \\
& & & & & & & \\
\hline
\end{tabular}




\section{Form analysis}

The different measures described below correspond to the blanks in the form analysis data sheet (Table $I$ ) used in the following section so that the reader can refer from one to the other. Thus, a measurement for the value "A-1," as described below, is entered in the space designated $A-1$ in the form analysis data sheet. The same code is also used to identify the corresponding measurements in Fig. 3.

\section{A. Vertical}

1. Posterior maxilla ( $P M$ vertical). Measure from $\mathrm{SE}$ point to $\mathrm{X}$ point (not to the functional occlusal plane). Record on the data form.

2. Anterior maxilla ( $A M$ vertical). Measure from the intersection of the AM and UM lines down to the functional occlusal plane (or to the neutral occlusal axis if the two coincide). If open-bite occurs at the premolars, the maxillary contact line is to be used, since a functional occlusal plane here does not exist as such. This measure represents the effective (not total) dimension of the anterior nasomaxillary complex. Record on the data sheet.

If any CF axis rotation has occurred, it will change the dimension above the UM-AM intersection point. However, the dimension as measured below this point also incorporates any influence of rotation on this effective vertical dimension.

3. Composite ramus-cranial floor (SO and Ra verticals). Measure from SO point down to the intersection of the Ra vertical plane and the functional occlusal plane. If open-bite occurs, measure to the mandibular contact line. Record. (Note: Determination of individual values for the SO and Ra verticals is not necessary for the present analysis. This should he done for cross-sectional studies, however, since it will show reciprocal adjustments between the two; that is, a "Iong" cranial base can accompany a "short" ramus in order to provide aggregate vertical balance.)

4. Functional occlusal plane divergence. If the functional and neutral occlusal lines do not coincide, measure the distance between them where each intersects (a) the Ra vertical line and (b) the $\mathrm{AM}$ vertical line. Record. These measurements indicate the actual extent of imbalance between them relative to these two vertical planes.

5. Maxillary-mandibular contact divergence. If any anterior premolar or posterior molar open-bite exists, measure from the maxillary to the mandibular contact lines along the $\mathrm{Ra}$ vertical plane. Record. This is the actual extent of imbalance between them relative to the ramus and also the amount of "adjustment" that would be required here to bring them into convergence relative to a functional ocelusal plane. A similar measurement may be taken, if desired, on the AM vertical line. The distance of either the maxillary or the mandibular contact lines from the neutral occlusal axis indicates the extent of imbalance, relative to this "ideal" plane, which has produced the degree of rotation observed.

6. Incisor relationships. If occlusal rotation has occurred, note whether or not the maxillary incisor meets or bypasses the functional occlusal plane to provide a 3 or $4 \mathrm{~mm}$. overlap with the mandibular incisor. If it does not, would 


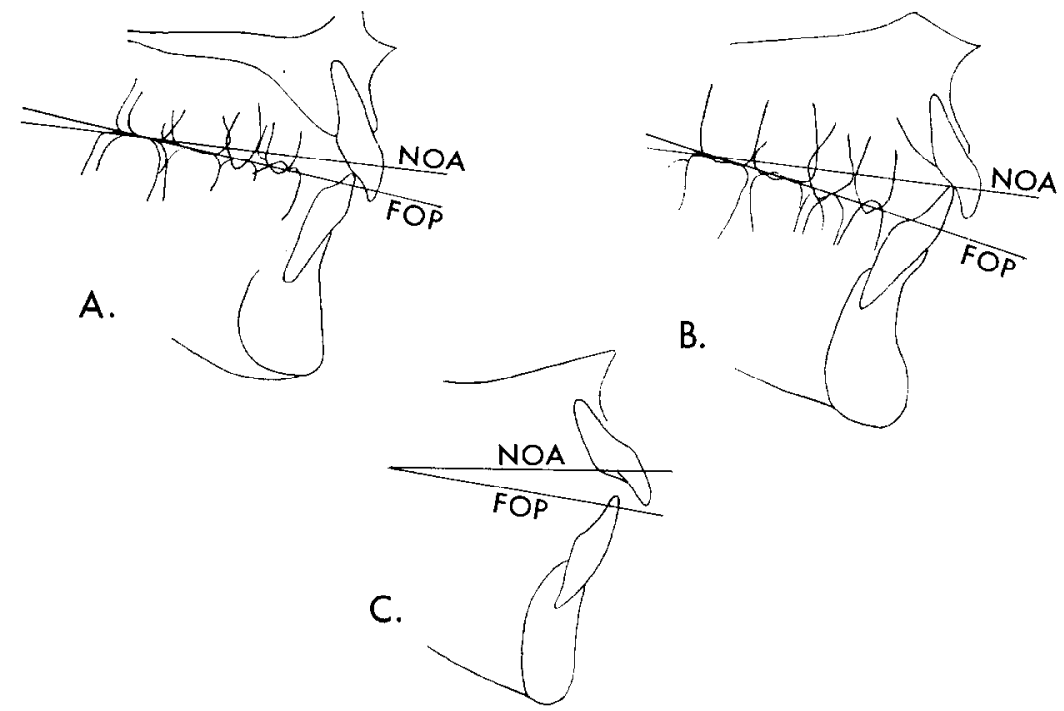

Fig. 4. Diagrams illustrating three possible incisor relationships associated with downward occlusal rotation from the neutral occlusal axis (NOA). In A, proper maxillarymandibular incisor overlap contact is present; note that the cusps meet and slightly bypass the functional occlusal plane (FOP). In B, the maxillary incisor has not met the functional occlusal plane. The cusp of the mandibular incisor, however, has markedly bypassed this plane and thereby provides "normal" overlap contact. Note that the cusp of this incisor is located well above the functional occlusal level common to the molars and premolars. In C, a simple backward tilt of the maxillary incisor would place it in a position corresponding to the pattern seen in A.

a simple lingual tipping be sufficient to place it in functional occlusion? If not, has the mandibular incisor bypassed the functional ocelusal plane sufficiently to provide normal overlap? (See Fig. 4.) Enter comment on the data sheet.

7. Compare and evaluate the measured values for 1, 2 and 3 above. If they exactly match, the functional and neutral occlusal planes coincide and these vertical dimensions (equivalents) are thus in balance with each other. If a dimensional imbalance exists, either an upward or (more commonly) a downward ocelusal rotation has occurred. The extent of this imbalance is indicated by $4 \mathrm{a}$ and $4 \mathrm{~b}$ above. If occlusal rotation has taken place in a uniform manner, openbite is not present. If a differential exists between them, however, the actual extent of maxillary-mandibular divergence is indicated by 5 above. Summarize the evaluation on the data sheet.

B. Horizontal

1. Nasomaxilla (at SPr). Measure from Pr to the PM vertical line parallel to the functional occlusal plane (or neutral occlusal axis if they both coincide or to the maxillary contact plane if premolar open-bite exists). Record.

2. Nasomaxilla (at $A$ point). Measure from A point to the PM vertical line parallel to the appropriate occlusal line. Record.

3. Cranial floor ( $S O$ horizontal). Measure from SO point to SE point. Record. This horizontal dimension represents the cranial floor, including its lateral 
portions overlying the mandibular ramus. Only the midline spheno-occipital part (cranial base), however, is shown in head film tracings.

4. Total the values for 1 and 3 above. Record.

5. Total the values for 2 and 3 above. Record.

6. Mandibular corpus (at IPr). Measure from inferior $\operatorname{Pr}$ to LT point parallel to the functional occlusal plane (or to the neutral occlusal axis if they both coincide or to the mandibular contact line if premolar open-bite occurs). Record.

7. Mandibular corpus (at $B$ point). Measure from $\mathbf{B}$ point to L'l point parallel to the appropriate ocelusal plane. Record.

8. Ramus. Measure from LT point to the Ra vertical line (not to the posterior border of the ramus) along the appropriate occlusal line or parallel to SO horizontal, as desired. Record.

9. T'otal the values for 6 and 8 above. Record.

10. Total the values for 7 and 8 above. Record.

11. Compare and evaluate the measured values: 1 with $6 ; 2$ with 7 ; 3 with $8 ; 4$ with 9 ; and 5 with 10 . If a significant dimensional imbalance occurs between any two of these individual sets of equivalents, does a reciprocal imbalance oceur between others which offsets the effect of imbalance and "adjusts" their aggregate dimensions? If not, analyze the nature of the imbalances present to ascertain which equivalents mismatch. Relate this to the nature of any skeletal problem, malocclusion, overbite, overjet, etc. that occurs as a consequence. (Note: Maxillary length, either as an individual or as an aggregate measure, may exceed mandibular length in an approximate 0 to $3 \mathrm{~mm}$. normal range. Within this range, dimensions may be regarded as "balanced.") Summarize evaluation on the data sheet.

\section{Growth analysis}

A. Vertical. Increments of growth are determined by subtracting the measured values for the various dimensions of the younger age from those at the older age. The itemization of the accompanying "growth" data sheet (Table II) corresponds to that of the "form" data sheet (Table I).

Analyze the meaning and significance of the different growth increments relative to the status of dimensional balance at the ages involved. If balance or near balance of form originally occurred, did the growth increments maintain this balance? If a dimensional imbalance existed at the younger age, did the increments of growth (1) improve them, (2) sustain them without material change, or (3) aggravate them?

B. Horizontal. Calculate the increments of growth from the previously measured values at the two age levels involved. Enter these values in the data form. Analyze and evaluate the balance of growth relative to the particular nature of the dimensions at these ages. Determine if increments (1) maintained an original balanced facial form, (2) sustained an imbalanced form, (3) improved an imbalanced form, or (4) aggravated an imbalanced form. Does aggregate balance of the growth increments between the different sets of 
Table II. Growth analysis

\begin{tabular}{|c|c|c|c|c|}
\hline & \multirow[b]{2}{*}{$\begin{array}{c}\text { Subject } A \\
\text { to } 14 \text { yrs. } \\
(m m .)\end{array} \mid$} & \multirow[b]{2}{*}{$\begin{array}{c}\text { Subject } B \\
\text { ry to } 18 \text { yrs. } \\
(m m .)\end{array}$} & \multicolumn{2}{|c|}{ Subject $C$} \\
\hline & & & $\begin{array}{l}8 \text { to } \\
14.6 \mathrm{yrs} \text {. } \\
(\mathrm{mm} .)\end{array}$ & $\begin{array}{l}10.1 \text { to } \\
12.6 \text { yrs. } \\
(\mathrm{mm} .)\end{array}$ \\
\hline \multicolumn{5}{|l|}{ A. Vertical } \\
\hline 1. Posterior maxilla (PM vertical) & 15.6 & 16.5 & 9.2 & 4.9 \\
\hline $\begin{array}{l}\text { 2. Anterior maxilla (AM-UM intersection to } \\
\text { functional occlusal plane) } \\
\text { 3. Composite ramus-cranial floor (Ra-SO } \\
\text { verticals) }\end{array}$ & 15.7 & 13.3 & 10.2 & 4.8 \\
\hline $\begin{array}{l}\text { a. cranial floor ( } \mathrm{SO} \text { vertical) if desired } \\
\text { b. ramus ( } \mathrm{Ra} \text { vertical) if desired }\end{array}$ & 12.9 & 19.9 & 9.1 & 5.6 \\
\hline $\begin{array}{l}\text { 4. Commentary on vertical incremental } \\
\text { balance relative to dimensional balance at } \\
\text { the two ages: (See later discussion) }\end{array}$ & & & & \\
\hline \multicolumn{5}{|l|}{ B. Horizontal } \\
\hline 1. Nasomaxilla (Pr to PM vertical) & 5.0 & 12.3 & 9.0 & 2.0 \\
\hline 2. Nasomaxilla (A point to PM vertical) & 2.7 & 9.7 & 5.9 & 2.0 \\
\hline 3. Cranial floor (SO horizontal) & 3.5 & 7.3 & 3.3 & 1.6 \\
\hline 4. Total $(1+3)$ & 8.5 & 20.6 & 12.3 & 3.6 \\
\hline 5. Total $(2+3)$ & 6.2 & 17.0 & 9.2 & 3.6 \\
\hline 6. Mandibular corpus (IPr to LT) & 5.3 & 16.4 & 7.9 & 0.0 \\
\hline 7. Mandibular corpus (B point to $\mathrm{LT}$ ) & 4.3 & 13.1 & 8.3 & -1.2 \\
\hline 8. Ramus (LT to Ra vertical) & 4.4 & 5.9 & 2.6 & 2.8 \\
\hline 9. Total $(6+8)$ & 9.7 & 22.3 & 10.5 & 2.8 \\
\hline 10. Total $(7+8)$ & 8.7 & 19.0 & 10.9 & 1.6 \\
\hline $\begin{array}{l}\text { 11. Commentary on horizontal incremental } \\
\text { balance relative to dimensional balunce at } \\
\text { the two ages: (See later discussion) }\end{array}$ & & & & \\
\hline
\end{tabular}

equivalents serve to compensate for any deficiency or excess in some individual dimension?

\section{Case examples}

Form and growth analyses of three different patients are presented to illustrate the procedure and to demonstrate cephalometric interpretations as based on the morphologic and morphogenic concepts utilized in this study. Only a few representative examples of the many possible combinations that occur in equivalent and aggregate balance can be shown. These cases have been selected primarily to illustrate the principles involved and not to classify the basic categories of form and growth that can be revealed by this method of analysis. The measured values for these case examples are given in Tables I and II. A brief description and an evaluation of each is presented below. The items in the "form" and the "growth" tables correspond to each other by number. They also correspond to the outline of procedure previously given and to the code used in Fig. 3. 


\section{Subject A (untreated)}

The craniofacial complex of this growing child demonstrates a reasonably close dimensional balance among the different horizontal and vertical equivalents as well as their aggregate composites (Fig. 5). The different growth increments between the two ages are similarly elose in balance, thereby maintaining the status of the form (dimensional) balance. Note that at the age of 14 years the last molar is not used to determine the occlusal plane, since it is situated well above the other molars and premolars.

Vertical. At 7 years, a differential between the three vertical composite equivalents (anterior and posterior nasomaxilla and cranial floor-ramus) produces a slight upward occlusal rotation relative to the neutral ocelusal axis. The extent of actual imbalance between the cranial floor-ramus and the posterior maxillary vertical
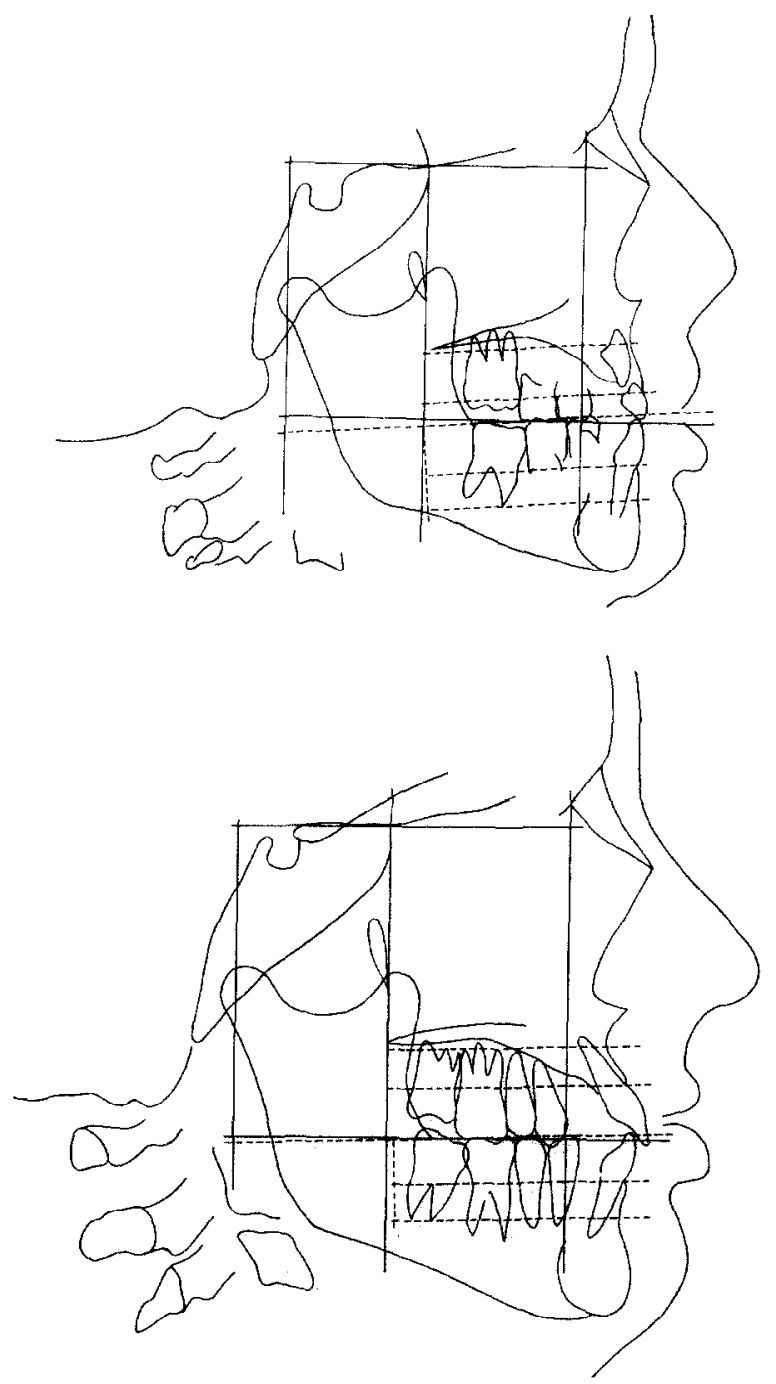

Fig. 5. Subject $\mathbf{A}$ at 7 and 14 years of age. (See text for discussion.) 
dimensions is $4.7 \mathrm{~mm}$., which indicates that the former is "too long" (or that the latter is "too short") by this amount, thereby resulting in an upward rotation. It is not possible to state which of these two dimensions is in actual imbalance, since their disproportions are relative to each other. Note that the subsequent "imbalanced" vertical growth increments have served to reduce (and to improve) the extent of upward rotation between the two ages to a $2.0 \mathrm{~mm}$. difference between the functional and neutral occlusal planes. This is regarded as a negligible degree of vertical imbalance and occlusal rotation. More important, the slight dimensional imbalance between the PM and SO-Ra vertical measurements is proportionate to a corresponding adjusted imbalance between the PM and AM. vertical measurements, thereby providing complete occlusion without open-bite or other major skeletal problems.

Horizontal. At both ages, the over-all length of the maxilla (at $\mathrm{Pr}$ ) is about $2 \mathrm{~mm}$. longer than the mandibular eorpus, which is within the normal range (although approaching maximum). Their respective balanced increments of growth serve to maintain this dimensional balance from the ages of 7 to 14 years. An excess of lengthening at mandibular $B$ point resulted in an imbalance relative to maxillary A point, thereby producing a slight Class 111 tendency at 14 years with regard to the "basal" portions of the maxilla and mandible. This is compensated in the maxilla, however, by maintaining proper incisor and alveolar protrusion ( $\operatorname{Pr}$ point). The "effective" horizontal dimensions of the cranial base and the ramus (direct equivalents to each other) are in close dimensional as well as incremental balance. The aggregate dimensions of the various equivalent composites are also balanced.

\section{Subject B (untreated)}

Vertical. At the age of 7 years, a vertical imbalance between the anterior maxilla, the posterior maxilla, and the vertical cranial floor-ramus has produced a significant degree of downward occlusal rotation (Fig. 6). The difference of $7.9 \mathrm{~mm}$. between the cranial floor-ramus vertical and the posterior maxillary vertical represents the actual amount by which the former would have to have lengthened (or the latter would need to be shortened) in order to align the functional occlusal plane with the "ideal" neutral occlusal axis. It is observed, however, that a proportionate imbalance has also occurred at the anterior maxillary plane, thereby providing functional occlusion at the premolar level, even though marked dimensional imbalances exist. The difference of $5.2 \mathrm{~mm}$. on the AM line represents the amount of imbalance that is involved in the anterior part of the maxilla relative to the functional and neutral occlusal planes. Note that a simple lingual tipping of the maxillary incisor would be sufficient to bring its free edge to a proper vertical level relative to the functional occlusal plane (Fig. 4). At 18 years, the degree of downward occlusal rotation has been much reduced by imbalanced growth increments at the three vertical planes. Thus, an actual imbalance of growth has served to improve an original dimensional imbalance. Had the growth increments themselves been "balanced," the dimensional disproportion would have been sustained without change (Fig. 1).

Horizontal. At 7 years of age, the length of the maxillary body greatly exceeds that of the mandibular corpus, both at the $\operatorname{Pr}$ and at the $A$ and $B$ points. Note that the cranial floor-ramus horizontal equivalents, however, are in near dimensional balance and that they therefore do not materially compensate or offset the disproportion of the maxillary-mandibular corpus equivalents. Aggregate dimensions are at least $5 \mathrm{~mm}$. out of balance beyond the normal maximum range.

At the age of 18 years, a significant and desirable "imbalance" of horizontal growth has occurred between the maxilla and the mandibular corpus. This has reduced the extent of dimensional disproportion between them. Thus, imbalanced growth changes have improved an original dimensional imbalance. Composite dimensions, however, are still about $2 \mathrm{~mm}$. out of aggregate balance beyond maximum latitude. 

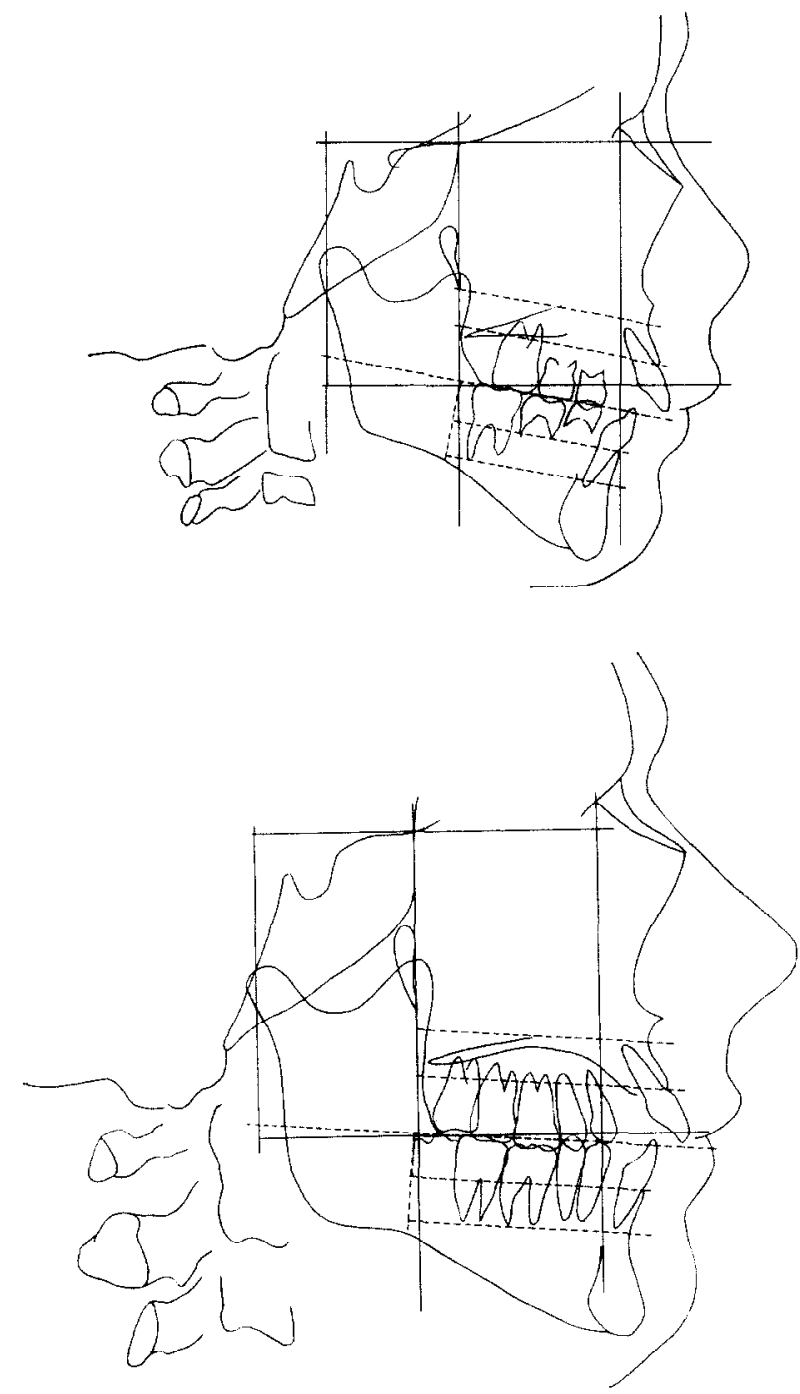

Fig. 6. Subject B at 7 and 18 years of age. (See text for discussion.)

\section{Subject C (untreated)}

Tabular data only are presented for this subject at the ages of 8 to 14.6 and 10.1 to 12.6 years. The purpose is to demonstrate the picture of a restricted 2-year growth increment as seen within a larger 6-year period. This subject also demonstrates the process of dimensional adjustment that provides adequate over-all balance, even though several regional equivalent imbalances exist. Further, this case shows the occurrence of age-related growth spurts and a factor of differential timing during growth changes among the various structural equivalents.

Vertical. A dimensional imbalance between the three composite vertical equiv. alents has produced a moderate downward ocelusal rotation relative to the neutral ocelusal axis. At 8 years, the cranial base-ramus vertical composite is $4.1 \mathrm{~mm}$. "too 
short" relative to the posterior nasomaxilla (or the maxilla is "too long" by a like amount). However, a proportionate vertical imbalance has also oceurred in the anterior maxilla, thereby providing an adjusted over-all occlusal relationship without open-bite at the premolar level. The increments of growth in these three vertical dimensions have sustained the degree of occlusal rotation without material change from 8 to 14.6 years of age. Note that a marked spurt in vertical growth, in contrast to the various horizontal increments, has occurred during the 10.1 to 12.6 period.

Horizontal. At 8 years of age, the length of the nasomaxilla, at both $\operatorname{Pr}$ and $\mathrm{A}$ point, is slightly excessive beyond the normal maximum range relative to mandibular corpus length. However, the horizontal ramus dimension compensates by exceeding its cranial floor equivalent by an amount that brings composite dimensions into nearly perfect aggregate balance. Were this not the case, excessive maxillary protrusion and a Class II occlusion would have resulted. Compare and contrast this situation with that previously deseribed for Subject B in which compensation between equivalents did not produce an adequate aggregate balance.

By the age of 10 years, an imbalance of growth has greatly increased the horizontal dimensional disproportion between the maxilla and the mandibular corpus. A corresponding imbalance between the ramus and the eranial base, however, proportionately offsets this situation so that composite horizontal dimensions retain an aggregate balance within the normal range. Between the ages of 10 and 12.6 years, continued growth by the maxillary horizontal together with a complete lack of growth by the mandibular corpus further increases the disproportion between them. Compensation by the ramus-cranial base equivalents still provides aggregate adjustment, however, although now at the maximum end of the normal range. Note that corpus length at $B$ point has actually decreased (this outer surface is resorptive).

At the age of 14.6 years, a marked spurt of differential ("imbalanced") growth by the mandibular corpus as measured at $B$ point finally brings it into good dimensional balance with the maxillary body at A point, thereby correcting the sizable imbalance that had occurred between them. Note that an actual decrease in the horizontal dimension of the ramus, which had been disproportionately large to compensate for the short corpus, occurs between 12 and 14 years. This change accompanies the relatively sudden increase in corpus length (the anterior ramus margin is resorptive and moves backward as the corpus elongates posteriorly). The other remaining horizontal equivalents maintain their original pattern by essentially balanced increments of growth. Thus, the maxilla at $\mathrm{SPr}$ is still excessive (by about $2 \mathrm{~mm}$.) in relation to the corpus at IPr, but a ramus-cranial floor imbalance continues to offset this differential so that an adequate aggregate balance is sustained among the various composites of equivalents.

\section{REFERENCES}

1. Bimler, H. P.: Roentgenoscopic method of analyzing the facial correlations, Tr. European Orthodont. 33: 241-253, 1957.

2. Bimler, H. P.: Facial pattern formula, Tr. European Orthodont. Soe. 36: 224-235, 1960.

3. Enlow, D. H.: The Human Face, New York, 1968. Hoeber Medical Division, Harper \& Row, Publishers.

4. Enlow, D. H., and Hunter, W. S.: The growth of the face in relation to the cranial base, Tr. Furopean Orthodont. Soc. In press.

5. Jenkins, D. H.: Analysis of orthodontic deformity employing lateral cephalostatic radiography, AM. J. ORThonontics 41: 442-452, 1955. 